\title{
Comment on Quantitative Evaluation of Osteocyte Morphology and Bone Anisotropic Extracellular Matrix in Rat Femur
}

\author{
Henrik Birkedal ${ }^{1}$ (D) \\ Received: 25 June 2021 / Accepted: 31 July 2021 / Published online: 10 August 2021 \\ (c) The Author(s), under exclusive licence to Springer Science+Business Media, LLC, part of Springer Nature 2021
}

\section{To the Editor,}

I read the recent paper by Ishimoto et al. [1] with interest. These authors correlate mineral orientation obtained from transmission X-ray diffraction with measures of osteocyte lacunar orientation from confocal laser scanning microscopy in rat femur. They proceed to make a model of mechanical adaption of osteocyte lacunae through their orientation to enhance sensing of fluid flow induced mechanical stimuli by osteocytes.

However, the authors overlook an important aspect of rodent long bone growth in their interpretation. As they state [1], rats do not undergo Haversian remodeling like for example humans do and the therefore lack secondary osteons. Rather, rat femora contain two types of bone: a central disorganized band formed by endochondral ossification in the growth plate as well as periosteal/endosteal bands of more organized appositional lamellar bone. In the central band, there are remnants of calcified cartilage clearly testament of the endochondral origin as shown first by us in Wistar rats [2] and very shortly thereafter in Rattus norvegicus by others [3]. Using synchrotron tomography, both we [4] and Shipov et al. [3] reported that the osteocyte lacunar density was increased in the central, more disorganized, endochondral band. Similar conclusions have been made for mouse bone $[5,6]$. Furthermore, osteocyte lacunae were more anisotropic in shape in the organized lamellar bone. The major orientation determinant of osteocyte lacunae in the lamellar bone was that the lacunar short axis be perpendicular to the femoral length direction [4]. This indicated that the orientation of osteocyte lacuna was primarily determined by the orientation and organization of mineralized collagen fibrils and by implication the associated mineral (see also discussion in reference [7]). This explains the observations reported by Ishimoto et al. [1] by the fact that two different mechanisms of bone growth are at play in rat long bones without the need to postulate an optimization towards fluid flow stress sensing that in my view would require lifting a rather higher burden of proof than the correlation provided by Ishimoto et al.

\section{Declarations}

Conflict of interest The author declares that he has no conflict of interest.

\section{References}

1. Ishimoto T, Kawahara K, Matsugaki A, Kamioka H, Nakano T (2021) Quantitative evaluation of osteocyte morphology and bone anisotropic extracellular matrix in rat femur. Calcif Tissue Int. https://doi.org/10.1007/s00223-021-00852-1

2. Bach-Gansmo FL et al (2013) Calcified cartilage islands in rat cortical bone. Calcif Tissue Int 92(4):330-338

3. Shipov A et al (2013) Unremodeled endochondral bone is a major architectural component of the cortical bone of the rat (Rattus norvegicus). J Struct Biol 183:132-140

4. Bach-Gansmo FL et al (2015) Osteocyte lacunar properties in rat cortical bone: differences between lamellar and central bone. $\mathrm{J}$ Struct Biol 191:59-67

5. Ip V et al (2016) Remnant woven bone and calcified cartilage in mouse bone: differences between ages/sex and effects on bone strength. PLoS ONE 11:e0166476

6. Wittig NK et al (2019) No signature of osteocytic osteolysis in lactating NMRI mice. Calcif Tissue Int 105:308-315

7. Grünewald TA et al (2020) Mapping the 3D orientation of nanocrystals and nanostructures in human bone: indications of novel structural features. Sci Adv 6:eaba4171

Publisher's Note Springer Nature remains neutral with regard to jurisdictional claims in published maps and institutional affiliations.

Henrik Birkedal

hbirkedal@chem.au.dk

1 Department of Chemistry and iNANO, Aarhus University, 14 Gustav Wieds Vej, 8000 Aarhus, Denmark 